\title{
GENERALIZED CYCLOTOMIC PERIODS
}

\author{
RONALD J. EVANS
}

\begin{abstract}
Let $n$ and $q$ be relatively prime integers with $n>1$, and set $N$ equal to twice the product of the distinct prime factors of $n$. Let $t(n)$ denote the order of $q$ $(\bmod n)$. Write $\eta=\Sigma_{v=0}^{(n)-1} a_{v} \zeta_{n}^{q^{\circ}}$, where $\zeta_{n}=\exp (2 \pi i / n)$. If $a_{v}=1$ for all $v$, then $\eta$ is Kummer's cyclotomic period, and if $a_{v}=\exp (2 \pi i v / t(n))$ for each $v$, then $\eta$ is a type of Lagrange resolvent. For certain classes of $a_{v} \in \mathbf{Q}\left(\zeta_{n}^{N}\right)$, necessary and sufficient conditions for the vanishing of $\eta$ are given, and the degree of $\eta$ over $\mathbf{Q}$ is determined.
\end{abstract}

1. Introduction and notation. Let $n$ and $q$ be fixed relatively prime integers with $n>1$, and set $N$ equal to twice the product of the distinct prime factors of $n$. Fix an integer $s$ prime to $n$ and set $K_{n}=K_{n, s}=\mathbf{Q}\left(\zeta_{s}, \zeta_{n}^{N}\right)$, where $\zeta_{n}=\exp (2 \pi i / n)$. For any integer $j$ prime to $n$, define $\sigma_{j} \in \operatorname{Gal}\left(\mathbf{Q}\left(\zeta_{n s}\right) / Q\left(\zeta_{s}\right)\right)$ by $\sigma_{j}\left(\zeta_{n}\right)=\zeta_{n}^{j}$. Let $t(n)$ denote the order of $q(\bmod n)$. Fix $a_{v} \in K_{n}(0<v<t(n))$ with not all $a_{v}$ vanishing, and define the generalized cyclotomic period $\eta$ by

$$
\eta=\sum_{v=0}^{t(n)-1} a_{v} \zeta_{n}^{q^{\circ}} .
$$

If all $a_{v}$ equal 1 , then $\eta$ is the cyclotomic period $\Sigma \zeta_{n}^{q^{\circ}}$ first studied for general $n$ by Kummer [2], but studied for prime $n$ over half a century earlier by Gauss. If $a_{v}=\exp (2 \pi i v / t(n))$ for each $v$, where $n$ is a prime power, then $\eta$ is a type of Lagrange resolvent studied in Weber's book [5, §19].

Fuchs [1] proved in essence the following facts about cyclotomic periods.

(1) If $t(n)=p t(n / p)$ for some prime $p$ dividing $n$, then $\Sigma \zeta_{n}^{q^{\circ}}=0$; and, conversely,

(2) if $\Sigma \zeta_{n}^{q^{\circ}}=0$, then $t(n)=p t(n / p)$ for some prime $p$ dividing $n$; and

(3) if $\Sigma \zeta_{n}^{q^{\circ}} \neq 0$, then $\Sigma \zeta_{n}^{q^{\circ}}$ has degree $\phi(n) / t(n)$ over $Q$, where $\phi$ is Euler's function. (Earlier, Kummer [2, p. 5] had stated (3) without proof.)

We prove here some analogues of Fuchs' results, for certain generalized periods $\eta=\Sigma a_{v} \zeta_{n}^{q^{\circ}}$ in place of $\Sigma \zeta_{n}^{q^{\circ}}$. In the process, we obtain simple new proofs of (1), (2), and (3).

In 1977, Kurt Mahler wanted to know when the period $\Sigma \zeta_{n}^{2^{\circ}}$ vanishes, in order to glean information about the behavior of the function $\sum_{v=0}^{\infty} z^{2^{\circ}}$ near the unit circle (see [4]). Seeking to answer his query, D. H. and E. Lehmer were led to rediscover (1) and (2) (see [3], but note that the formulation given there is not quite correct).

Received by the editors February 14, 1980 and, in revised form, April 3, 1980. Presented at the Western Number Theory Conference, Asilomar, December 20, 1979.

AMS (MOS) subject classifications (1970). Primary 10G05.

$K e y$ words and phrases. Cyclotomic periods. 
From here on, write $n=p^{\alpha} m$, where $p$ is prime, $p \nmid m$, and $\alpha>1$.

\section{Generalization of (1).}

TheOREM 1. Assume that $a_{v}=\sigma_{q}\left(a_{0}\right)$ for each $v$, and suppose that $t(n)=p t(n / p)$ for some $p$ dividing $n$. Then $\eta=0$.

Proof. Let $w=t(n / p)$. We have

$$
\eta=\sum_{v=0}^{t(n)-1} \sigma_{q^{\circ}}\left(a_{0}\right) \zeta_{n}^{q^{\circ}}=\sum_{u=0}^{w-1} \sigma_{q^{\mu}}\left\{\sum_{x=0}^{p-1} \sigma_{q^{w x}}\left(a_{0} \zeta_{n}\right)\right\} .
$$

Now, $q^{w}=1+j n / p$ for some $j$ prime to $p$. Since $q^{w p} \equiv 1(\bmod n)$, we see that $p$ divides $n / p$, so $q^{w x} \equiv 1+x j n / p(\bmod n)$ for $0<x<p$. Thus, since $a_{0} \in K_{n}$, $\sigma_{q \times x}\left(a_{0}\right)=a_{0}$. Therefore

$$
\eta=\sum_{u=0}^{w-1} \sigma_{q^{\mu}}\left\{a_{0} \zeta_{n} \sum_{x=0}^{p-1} \zeta_{p}^{x j}\right\}=0
$$

since the inner sum vanishes. Q.E.D.

TheOREM 2. Assume that $a_{v}=\varepsilon^{v}$ for each $v$, where $\varepsilon$ is a $t(n)$ th root of unity. Suppose that $t(n)=p w$ for some prime $p$ dividing $n$, where $w=t(n / p)$. Write $\varepsilon^{w}=\zeta_{p}^{k}$ and $q^{w}=1+j n / p$. Suppose further that $-k / j(\bmod p)$ is not congruent to a power of $q(\bmod p)\left(\right.$ this holds, for example, if $\left.\varepsilon^{w}=1\right)$. Then $\eta=0$.

Proof. The argument is essentially the same as that for Theorem 1. Q.E.D.

Theorem 2 proves one direction of Weber's theorem $[5, \S 19]$ while Lemma 5 (below) proves the other direction

Note that if $a_{0}=\varepsilon=1$, then Theorems 1 and 2 reduce to (1).

\section{Generalization of (2). •}

LEMMA 3. We have $t(n) \neq p t(n / p)$ if and only if either $t(n)=t(m p)$ or

$$
p^{\alpha}=2^{\alpha}>8, \quad t(n)=2 t(m), \quad 2 \nmid t(m), \quad \text { and } q \equiv 3(\bmod 4) .
$$

Proof. If $t(n)=t(m p)$ or (4) holds, clearly $t(n) \neq p t(n / p)$. Conversely, suppose that $t(n) \neq p t(n / p)$ and $t(n) \neq t(m p)$. We must prove (4). Note that $p^{A} \|\left(q^{t(m p)}-1\right)$ for some $A$ with $1<A<\alpha$. Suppose that $p$ is odd. Then $p^{A+B} \|\left(q^{p^{2}(m p)}-1\right)$ for each $B>0$. Thus $t\left(m p^{A+B}\right)=p^{B} t(m p)$ for each $B>0$. Therefore, $t(n)=p t(n / p)$, a contradiction. Thus $p=2$. We can now obtain a contradiction exactly as before, unless $A=1$ and $t(n)=2 t(2 m)$. These last equalities are easily seen to imply (4). Q.E.D.

LEMMA 4. Suppose that $p$ is the largest prime factor of $n$, and $t\left(p^{\alpha}\right)=p t\left(p^{\alpha-1}\right)$. Then $t(n)=p t(n / p)$.

Proof. If $n=p^{\alpha}$, the result is obvious, so we may assume that $p$ is odd. Assume that $t(n)=t(m p)$. Then $t\left(p^{\alpha}\right) \mid t(m p)$, so by hypothesis, $p \mid t(m p)$. Thus $p \mid \phi(m p)$, a contradiction. Therefore $t(n) \neq t(m p)$. Since also $p$ is odd, the result follows from Lemma 3. Q.E.D.

LeMMA 5. Suppose that $n=p^{\alpha}$ and $\eta=0$. Then $t(n)=p t(n / p)$. 
Proof. Assume that $t(n) \neq p t(n / p)$. Then by Lemma 3, either $t(n)=t(p)$ or $8 \mid n$, $t(n)=2, q \equiv 3(\bmod 4)$. In the latter event, $0=\eta=a_{0} \zeta_{n}+a_{1} \zeta_{n}^{q}$, so $\zeta_{n}^{q-1} \in K_{n}$, which is impossible because $2 \|(q-1)$. Thus $t(n)=t(p)$. Since $\eta=0, t(n)>1$. Thus $p>2$. Let $r_{v}$ denote the least positive residue of $q^{0}(\bmod p)$. Then

$$
0=\eta=\sum_{v=0}^{t(p)-1}\left(a_{v} \zeta_{n}^{q^{0}-r_{0}}\right) \zeta_{n}^{r_{0}}
$$

and the expressions in parentheses are in $K_{n}$. Since the elements $\zeta_{n}^{r_{0}}(0 \leqslant v<t(p))$ are distinct elements of a basis for $K_{n}\left(\zeta_{n}\right)$ over $K_{n}$, it follows that all $a_{v}$ vanish, a contradiction. Q.E.D.

Lemma 6. Write $z=t\left(p^{\alpha}\right), Q=q^{z}$, and let $T(m)$ denote the order of $Q(\bmod m)$. Then

$$
\sigma_{m+p^{\alpha}}(\eta)=\sum_{u=0}^{z-1} \sigma_{q^{\alpha}}\left(\delta_{u}\right) \zeta_{p^{\alpha}}^{q^{\mu}}
$$

where

$$
\begin{aligned}
\delta_{u} & =\sum_{x=0}^{T(m)-1} \theta_{x, u} \zeta_{m}^{Q^{x}}, \\
\theta_{x, u} & =\sigma_{m+p^{\alpha}} \sigma_{q^{u}}^{-1}\left(a_{z x+u}\right) .
\end{aligned}
$$

Proof. Since $T(m)=t(n) / z$ and $\zeta_{p^{\alpha}}^{Q}=\zeta_{p^{\alpha}}$,

$$
\sigma_{m+p^{\alpha}}(\eta)=\sum_{v=0}^{t(n)-1} \sigma_{m+p^{\alpha}}\left(a_{v}\right) \zeta_{m}^{q^{\alpha} \xi_{p^{\alpha}}^{q^{\alpha}}}=\sum_{u=0}^{z-1} \sum_{x=0}^{T(m)-1} \sigma_{m+p^{\alpha}}\left(a_{z x+u}\right) \zeta_{m}^{Q^{\alpha} q^{\alpha}} \zeta_{p^{\alpha}}^{q^{\alpha}},
$$

and the result follows. Q.E.D.

TheORem 7. Suppose that $\eta=0$. Then $t(n)=p t(n / p)$ for some $p$ dividing $n$.

Proof. The notation of Lemma 6 will be used here. We induct on the number of distinct prime factors of $n$. The induction starts by Lemma 5 , so it can be assumed that $n$ is not a prime power. Let $p$ be the largest prime factor of $n$. First suppose that $\delta_{u} \neq 0$ for some $u$. By (6) and (7), $\sigma_{q^{u}}\left(\delta_{u}\right) \in K_{p^{a}, m s}$. Since the left side of (5) vanishes, we may apply Lemma 5 to the generalized period on the right side of (5) to conclude that $t\left(p^{\alpha}\right)=p t\left(p^{\alpha-1}\right)$. Thus $t(n)=p t(n / p)$ by Lemma 4. Finally, suppose that $\delta_{u}=0$ for each $u$. Fix $u$ such that $\theta_{x, u} \neq 0$ some for $x$ (this is possible by (7)). All of the $\theta_{x, u}$ are in $K_{m, s p}$, so by (6) and the induction hypothesis, $T(m)=r T(m / r)$ for some prime $r$ dividing $m$. Multiplying by $t\left(p^{\alpha}\right)$, we find that $t(n)=r t(n / r)$. Q.E.D.

REMARK. If $n=m p^{\alpha}$ with $\alpha>1$ and $L=Q\left(\zeta_{n s}^{p}\right)$, then $\eta=0$ if and only if $\eta \in L$. Assume for the purpose of contradiction that $0 \neq \eta \in L$. By (5), $\zeta_{p}$ is a zero of a polynomial $f(x) \in L[x]$ with $f(0) \neq 0$ but with all of $f$ 's nonconstant monomials possessing the form $b x^{r}$, where $b \in L, p \nmid r$. Since $g(x)=x^{p}-\zeta_{p^{a-1}}$ is the minimal polynomial of $\zeta_{p}$ over $L, g(x) h(x)=f(x)$ for some $h(x) \in L[x]$. Since $h(0) \neq 0$, this implies that $f(x)$ has a monomial of the form $b x^{r}$ with $p \mid r$, a contradiction. 


\section{Generalization of (3).}

THEOREM 8. Suppose that $a_{v}=\sigma_{q^{\circ}}\left(a_{0}\right)$ for all $v$. If $\eta \neq 0$, then $\eta$ has degree $\phi(n) / t(n)$ over $\mathbf{Q}\left(\zeta_{s}\right)$.

Proof. It suffices to show that if $0 \neq \eta=\sigma_{c}(\eta)$, then $c \equiv$ power of $q(\bmod n)$. We will prove this by induction on the number of distinct prime factors of $\boldsymbol{n}$.

Suppose that $0 \neq \eta=\sigma_{c}(\eta)$. By Theorem $1, t(n) \neq p t(n / p)$, for each prime $p$ dividing $n$. Let $p$ now denote the largest prime factor of $n$. By Lemma 4, $t\left(p^{\alpha}\right) \neq p t\left(p^{\alpha-1}\right)$, so by Lemma 3 (with $p^{\alpha}$ in place of $n$ ), either

$$
t\left(p^{\alpha}\right)=t(p)
$$

or

$$
n=p^{\alpha}=2^{\alpha}>8, \quad t\left(2^{\alpha}\right)=2, \quad q \equiv 3(\bmod 4) .
$$

Suppose first that (9) holds. Then $a_{0} \zeta_{n}+a_{1} \zeta_{n}^{q}=\sigma_{c}\left(a_{0}\right) \zeta_{n}^{c}+\sigma_{c}\left(a_{1}\right) \zeta_{n}^{c q}$, so

$$
\left\{a_{0}-\sigma_{c}\left(a_{0}\right) \zeta_{n}^{c-1}\right\}=\zeta_{n}^{q-1}\left\{\sigma_{c}\left(a_{1}\right) \zeta_{n}^{q(c-1)}-a_{1}\right\} \text {. }
$$

We may assume without loss of generality that $c \equiv 1(\bmod 4)$, otherwise $c$ can be replaced by $c q$. Thus, the braced expressions in (10) are in $K_{n}$, and since $\zeta_{n}^{q-1} \notin K_{n}$ by (9), it follows that the left side of (10) vanishes. Thus

$$
\sigma_{c}\left(a_{0}\right)=a_{0} \zeta_{n}^{1-c},
$$

and by repeated applications of $\sigma_{c}$ to (11), we obtain

$$
\sigma_{c^{e}}\left(a_{0}\right)=a_{0} \zeta_{n}^{1-c^{*}} \quad(e>0) .
$$

If $c=1$, the result follows, so assume that $c \neq 1$. Then $2^{B} \|(c-1)$ for some $B>2$. Assume for the purpose of contradiction that $B<\alpha$. For each $A>0$,

$$
2^{B+A} \|\left(c^{2^{A}}-1\right) \text {. }
$$

Let $A=\alpha-1-B$. Then by (13) and (12) with $e=2^{A}$, we obtain $a_{0}=-a_{0}$, which contradicts the fact that $\eta \neq 0$. Thus $B>\alpha$, which yields the desired result.

Now suppose that (8) holds. Say $p=2$. Then the equality $\eta=\sigma_{c}(\eta)$ becomes $a_{0} \zeta_{n}=\sigma_{c}\left(a_{0}\right) \zeta_{n}^{c}$. Thus (11) holds and $c \equiv 1(\bmod 4)$, so that the desired result follows as above. It remains to consider the case $p>2$.

By (8), the right side of (5) is

$$
R=\sum_{u=0}^{t(p)-1} \sigma_{q^{u}}(\delta) \zeta_{p^{\alpha}}^{q^{\alpha}},
$$

where

$$
\delta=\sum_{x=0}^{T(m)-1} \sigma_{Q^{x}}\left(b_{0}\right) \zeta_{m}^{Q^{x}}, \quad b_{0}=\sigma_{m+p^{a}}\left(a_{0}\right) .
$$

Since $0 \neq \eta=\sigma_{c}(\eta)$, it follows from (5) that $0 \neq R=\sigma_{c}(R)$ (and in particular, $\delta \neq 0)$. For $0<u<t(p)$, write

$$
q^{u}=p s_{u}+r_{u}, \quad c q^{u}=p s_{u}^{\prime}+r_{u}^{\prime} \quad\left(0<r_{u}, r_{u}^{\prime}<p\right) .
$$

Clearly the $r_{u}$ are distinct and the $r_{u}^{\prime}$ are distinct. 
We have

$$
\sum_{u=0}^{t(p)-1}\left(\sigma_{q^{u}}(\delta) \zeta_{p^{\alpha}-1}^{S_{\alpha^{u}}}\right) \zeta_{p^{\alpha_{u}}}^{r_{u}}=R=\sigma_{c}(R)=\sum_{u=0}^{t(p)-1}\left(\sigma_{c q^{\mu}}(\delta) \zeta_{p^{\alpha-1}}^{s^{\prime}}\right) \zeta_{p^{\alpha}}^{r_{u}^{\prime}} .
$$

Since the elements $\zeta_{p^{\alpha}}^{1}, \ldots, \zeta_{p^{\alpha}}^{p-1}$ are linearly independent over $\mathbf{Q}\left(\zeta_{n s}^{p}\right)$, there exists a fixed value of $u$ such that

$$
r_{u}^{\prime}=r_{0}=1
$$

and

$$
\delta=\delta \zeta_{p^{\alpha-1}}^{s_{0}}=\sigma_{c q^{\alpha}}(\delta) \zeta_{p^{\alpha-1}}^{s^{\prime}}
$$

Thus, by (14) and (15),

$$
d=c q^{u}=1-p y
$$

and

$$
\sigma_{d}(\delta)=\delta \zeta_{p^{\alpha-1}}^{y}
$$

where $y=-s_{u}^{\prime}$. If $d=1$, the result follows, so assume that $d \neq 1$. Repeated applications of $\sigma_{d}$ to (17) yield

$$
\sigma_{d^{e}}(\delta)=\delta \zeta_{p^{\alpha-1}}^{y\left(d^{e}-1\right) /(d-1)} \quad(e>0) .
$$

We have $p^{B} \|(d-1)$ for some $B>1$. Thus, by (16),

$$
p^{B-1} \| y \text {. }
$$

Assume for the purpose of contradiction that $B<\alpha$. For each $A>0$,

$$
p^{A+B} \|\left(d^{p^{1}}-1\right)
$$

Let $A=\alpha-1-B$. By (20) and (18) with $e=p^{A}, p^{\alpha-1} \mid y\left(d^{p^{A}}-1\right) /(d-1)$, so by (19), $p^{\alpha} \mid\left(d^{p^{A}}-1\right)$, which contradicts (20). Thus $B>\alpha$, so

$$
d \equiv 1\left(\bmod p^{\alpha}\right)
$$

This completes the proof if $n$ is a prime power, i.e., if $m=1$. Thus assume that $m>1$. By (19), $p^{\alpha-1} \mid y$, so by (17),

$$
\sigma_{d}(\delta)=\delta \text {. }
$$

Since $\delta$ equals the generalized period $\Sigma_{x=0}^{T(m)-1} \sigma_{Q^{x}}\left(b_{0}\right) \zeta_{m}^{Q^{x}}$ with $0 \neq b_{0} \in K_{m, p^{\alpha}}$ and $\sigma_{Q^{x}} \in \operatorname{Gal}\left(Q\left(\zeta_{m s p^{\alpha}}\right) / Q\left(\zeta_{s p^{\alpha}}\right)\right)$, it follows from (21), (22), and the induction hypothesis that $d \equiv Q^{h}(\bmod m)$ for some $h$. Since $Q \equiv 1\left(\bmod p^{\alpha}\right),(21)$ yields $d \equiv Q^{h}$ $\left(\bmod p^{\alpha}\right)$. Thus $d \equiv Q^{h}(\bmod n)$ and $c \equiv$ power of $q(\bmod n)$. Q.E.D.

Theorem 8 states that if $L$ is a field with $\mathbf{Q}\left(\zeta_{s n}\right) \supset L \supset \mathbf{Q}\left(\zeta_{s}\right)$ such that $\mathbf{Q}\left(\zeta_{s n}\right)$ is cyclic over $L$, then the degree of $\operatorname{Tr}\left(a_{0} \zeta_{n}\right)$ over $\mathbf{Q}\left(\zeta_{s}\right)$ is either 0 or $\left|L: \mathbf{Q}\left(\zeta_{s}\right)\right|$, where Tr denotes the trace map from $Q\left(\zeta_{s n}\right)$ to $L$. In other words, if $\operatorname{Tr}\left(a_{0} \zeta_{n}\right)$ is nonzero, then it has the maximum possible degree over $Q\left(\zeta_{s}\right)$ that any element of $L$ can have. A modification of the proof of Theorem 8 shows that the hypothesis that $\mathbf{Q}\left(\zeta_{s n}\right) / L$ is cyclic may be dropped.

The author is very grateful to the Lehmers for their encouragement and helpful comments. 


\section{REFERENCES}

1. L. Fuchs, Ueber die Perioden, welche aus den Wurzeln der Gleichung $\omega^{n}=1$ gebildet sind, wenn $n$ eine zusammengesetzte Zahl ist, J. Reine Angew. Math. 61 (1863), 374-386.

2. E. Kummer, Theorie der idealen Primfaktoren der complexen Zahlen, welche aus den Wurzeln der Gleichung $\omega^{n}=1$ gebildet sind, wenn $n$ eine zusammengesetzte Zahl ist, Math. Abh. Kon. Akad. Wiss. Berlin (1856), 1-47; Collected Papers, vol. 1, Springer-Verlag, Berlin and New York, 1975, pp. 583-629.

3. D. H. Lehmer and E. Lehmer, Notices Amer. Math. Soc. 25 (1978), 145.

4. K. Mahler, On a special function, J. Number Theory 12 (1980), 20-26.

5. H. Weber, Lehrbuch der Algebra, 3rd ed., vol. 2, Chelsea, New York, 1961.

Department of Mathematics, University of California-San Diego, la Jolla, California 92093 